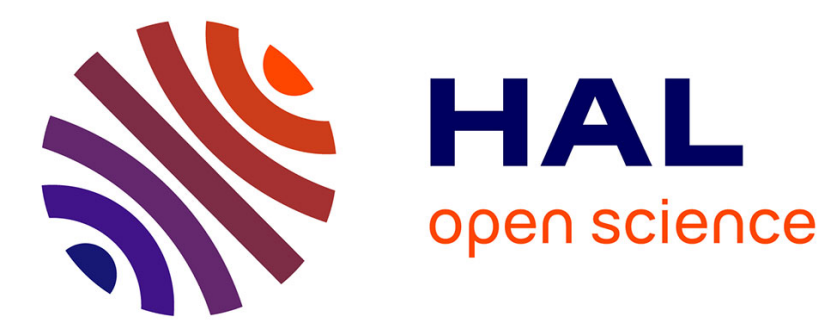

\title{
Histoire accélérée et générations historiques
}

Monica Heintz

\section{To cite this version:}

Monica Heintz. Histoire accélérée et générations historiques: Le cas contrasté de la Moldavie et de la Roumanie (années 2000). Ethnologie française, 2014, 44 (3), pp.399-407. 10.3917/ethn.143.0399 . hal-01610226

\section{HAL Id: hal-01610226 \\ https://hal.parisnanterre.fr/hal-01610226}

Submitted on 1 Jul 2020

HAL is a multi-disciplinary open access archive for the deposit and dissemination of scientific research documents, whether they are published or not. The documents may come from teaching and research institutions in France or abroad, or from public or private research centers.
L'archive ouverte pluridisciplinaire HAL, est destinée au dépôt et à la diffusion de documents scientifiques de niveau recherche, publiés ou non, émanant des établissements d'enseignement et de recherche français ou étrangers, des laboratoires publics ou privés. 


\title{
Histoire accélérée et générations historiques Le cas contrasté de la Moldavie et de la Roumanie (années 2000)
}

Monica Heintz

Laboratoire d'ethnologie et de sociologie comparative

\author{
RÉSUMÉ \\ Mots-clés : Génération. Europe de l'Est. Travail. Temporalités. Terrain miné. \\ Monica Heintz \\ Université de Paris Ouest Nanterre La Défense \\ Laboratoire d'ethnologie et de sociologie comparative (UMR CNRS 7186) \\ Maison Archéologie \& Ethnologie - René Ginouvès \\ 21, allée de l'Université \\ 92023 Nanterre cedex \\ monica.heintz@u-paris10.fr
}

Les changements rapides induits par les révolutions de 1989 ont laissé des marques durables parmi les populations actuelles d'Europe de l'Est. Étant divisées en générations historiques dont le passé et le futur ne se présentent pas sous le même angle, des " générations perdues " aux " générations attendues ", leur perception du social et l'inscription dans l'histoire qui les caractérisent se reflètent dans leurs choix économiques, politiques et culturels. Comment l'anthropologue peut-il ethnographier la diversité actuelle pour laquelle le vécu, auquel il n'a que partiellement accès, est déterminant ? Cette question est abordée à travers deux enquêtes conduites l'une en Moldavie, l'autre en Roumanie dans les années 2000.

Alors que des paramètres d'identification démographique tels que le genre, la classe sociale, le sexe ou même la race ont été à l'honneur dans les études ethnographiques des sociétés complexes, le paramètre "âge " est souvent négligé. "Dans l'éventail de modes de "sociation" mis en œuvre par l'être humain, les critères d'âge et de génération n'ont pas encore reçu toute l'attention qu'ils méritent " écrit Anne-Marie Peatrik dans un bilan anthropologique sur les générations comme facteurs d'organisation sociale [Peatrik, 1995]. Des efforts sont déployés depuis une vingtaine d'année pour la révélation de ces facteurs générationnels à travers des analyses des classes d'âge dans les sociétés africaines [Peatrik, 2000] ou dans les études de parenté dans les sociétés occidentales [Attias-Donfut et Segalen, 2007]. Dans ces études la mise en relief de structures pérennes (la définition même de la catégorie "grand-parent" se faisant en référence à la structure familiale) prime sur l'ancrage du phénomène générationnel dans l'histoire. En raison de l'accélération de l'histoire consécutive à chute du bloc communiste, dans les sociétés d'Europe de l'Est au tournant du XXI ${ }^{\mathrm{e}}$ siècle, l'âge et les clivages générationnels apparaissent comme des facteurs sociologiques déterminants. La radicalité des différences au sein de la population et des familles est révélatrice du poids historique de ces événements qui a créé des générations historiques solides dont l'identité se révèle aujourd'hui dans leur comportement politique, économique et social. Chacune d'entre elles a une mémoire et une perception du futur distincte de celle des autres, et donc un éventail de choix de vie, de style de vie, et même une conscience d'appartenance générationnelle propre.

Si les générations historiques sont en général liées à un événement ponctuel ou de courte durée, tel ne semble pas être le cas en Europe de l'Est où, plus que la rupture de 1989, c'est la période d'histoire accélérée post-1989 (dite "période de transition ») qui a forgé le clivage générationnel. Le terme d' "histoire accélérée " apparaît dans une collection d'articles sur l'Europe de l'Est publiée par Chris Hann [1994]. À considérer les témoignages qui y sont présentés, la perception de "l'accélération " des événements est une appréciation de ceux qui subissent les changements et n'ont pas la capacité de s'adapter ou de comprendre le sens de ces événements en raison de la rapidité avec laquelle ils 
s'enchaînent. Il n'est dès lors pas étonnant que l'âge constitue un facteur différenciateur par rapport à l'adaptabilité au nouveau régime social et politique. Et pourtant, la plupart des études postsocialistes insistent sur le poids de "l'héritage " du passé comme facteur explicatif principal [Burowoy and Verdery, 1999] - héritage plus lourd pour les moins jeunes - et non sur la différence d'adaptation au changement due à un ancrage sociologique plus important avec l'accroissement en âge, les plus vieux ayant plus de charges, plus de responsabilités et plus d'aversion pour le risque.

Sans nier l'importance de l'observation du changement social à une échelle historique plus large, c'est l'expérience que fait l'individu de l'accélération du temps lors de transformations rapides et de ses choix que je souhaite aborder dans cet article. En effet, avant d'avoir le recul historique nécessaire pour comprendre "le changement", chacun perçoit uniquement que le temps s'accélère - ou plus précisément que les événements se succèdent plus rapidement -, sans arriver à saisir leur sens. Ses comportements, choix, réactions se font alors dans un horizon temporel limité dont la planification à long terme est exclue, puisque la prévision semble impossible. Or, la planification et la prévision ne sont pas dans la même mesure l'apanage de tous les âges, car leur nécessité augmente en même temps que s'accroissent les obligations sociales. C'est pourquoi des individus d'âge différent ne sont pas égaux, ni face à la perception de l'accélération de l'histoire, ni dans leur capacité à la subir - et, partant, n'en font pas le même récit. Être sensible aux multiples réponses au changement provenant des membres de différentes générations devient alors indispensable pour saisir la complexité de la réalité sociale.

Je souhaite questionner dans cet article la manière dont un anthropologue collecte et analyse des données dans des sociétés connaissant une histoire accélérée, à travers la comparaison entre deux expériences de terrains est-européens marqués par des ruptures historiques majeures. Les deux études se fondent sur un engagement de longue durée, mais ont été menées dans des rapports au terrain différents quant au partage du vécu et aux engagements présents et futurs entre observateur et observés.

Le premier cas est celui de la Moldavie rurale post-1991, où j'ai mené une étude en 2003-2004 dans une période de confrontation politique entre différentes générations, mais qui n'entraînait pas à l'époque des changements politiques visibles. Malgré une connaissance active de la langue roumaine pratiquée dans la communauté étudiée et le partage d'un passé identitaire commun, j'étais sur le terrain dans une position d'extériorité par rapport à cette communauté, comme n'importe quel ethnographe, car je n'avais pas traversé les événements clés des dernières années.

Le second cas présenté est celui de la Roumanie urbaine où mes études s'étalent sur les quinze dernières années et dont le terrain relève de l'anthropologie du chez soi, puisque je partage le passé et la projection dans le futur de mes interlocuteurs. Dans les deux cas, le poids des facteurs "âge " et "génération " tels que définis dans le contexte post-événements de 1989-1991 est temporaire, et s'effacera probablement quand la capacité structurante de ces événements s'effacera.

En comparant la nature des données recueillies, il apparait que le lien que tisse l'ethnographe avec les différentes générations, ce qu'il partage, ce qui le lie comme ce qu'il renie, est le catalyseur des données qu'il obtient, des interprétations qu'il propose et des engagements qu'il prend. Le parallèle entre ces deux cas m'oblige à porter un regard réflexif sur le rôle des temporalités partagées entre observateur et observés dans la collecte des données de terrain et dans l'analyse ethnographique. Le contexte de l'histoire accélérée sert ici de loupe permettant de magnifier un phénomène présent dans toute rencontre ethnographique.

\section{" Ne volez pas notre futur": les conflits générationnels en République de Moldavie}

La République de Moldavie actuelle (qui recouvre la majeure partie de la région de Bessarabie) a été partie intégrante de la principauté roumaine de Moldavie jusqu'en 1812, date à laquelle l'empire tsariste incorpore la partie orientale de la Moldavie historique à la suite de la défaite de l'empire ottoman jusqu'alors principauté vassale. Celle-ci demeure au sein de l'empire tsariste jusqu'à la fin de la Première Guerre mondiale (27 mars 1918), puis s'unit avec la Roumanie, nouvel État-nation né entretemps (24 janvier 1859) de l'unification de deux principautés roumaines: la Moldavie et la Valachie. Après plusieurs changements d'autorité gouvernementale durant la Seconde Guerre mondiale, la région orientale de la Moldavie est incorporée à l'urss et forme ensemble avec la région de Transnistrie, la République Soviétique Socialiste 
Moldave (RSSM). En 1991, à la suite du démantèlement de l'Urss, elle déclare son indépendance et devient pour la première fois un état souverain sous le nom "République de Moldavie ». Parallèlement à ces changements institutionnels, la majorité roumanophone du pays (78\% de la population en 2003) se mobilise en 1989 contre la russification subie sous la république soviétique; elle réclame le droit à sa propre langue, qu'elle souhaite pouvoir appeler "roumain " et non plus "moldave », comme Staline l'avait imposé après la Seconde Guerre mondiale pour éviter les réclamations territoriales de la Roumanie, écrire en caractère latins et non plus cyrilliques. Elle réclame aussi le droit au respect de sa propre histoire, de sa culture, des traditions roumaines. L'indépendance acquise en 1991 à la fois confirme les droits réclamés en 1989 par les roumanophones et met l'État nouvellement créé dans une position d'illégitimité nationale. En effet, l'existence de la Roumanie voisine, qui affiche les mêmes langues, symboles, et histoire, puis le conflit ouvert avec une partie du territoire moldave, la Transnistrie, qui fait sécession, arrivent sur un fond de détresse économique due au brusque sevrage des relations avec la Russie. L'objectif de tous les gouvernements qui se succèdent depuis 1991 est alors de légitimer l'État nouvellement créé aux yeux d'une population à la fois nationaliste et multi-ethnique, dans un contexte d'appauvrissement croissant dû à la transition économique.

Ce récit succinct de l'histoire complexe de la République de Moldavie laisse deviner pourquoi en arrivant sur le terrain en octobre 2003 avec les connaissances historiques habituelles qu'acquiert un anthropologue dans la période préparatoire à travers la consultation des sources bibliographiques étrangères, $j$ 'ai senti que les faux pas me guettaient: comme le dit Dionigi Albera «le positionnement du chercheur est problématique, dans cette situation mouvante, où les terrains anciens se transforment, où en apparaissent de nouveaux, et où les conditions de la recherche évoluent rapidement " [Albera, 2001: 11]. J'avais pour objectif d'étudier l'éducation morale et religieuse des roumanophones orthodoxes en milieu rural, un sujet qui ne devait a priori susciter que des émotions positives chez mes interlocuteurs dans le contexte de renouveau religieux, mais les références implicites au passé soviétique de répression religieuse jetaient mes questions en terrain miné [ibid.]. Après seulement quelques jours dans un village situé au nord de la République, je me suis replongée dans une histoire plus détaillée que je venais de trouver à Chisinau [Fruntasu, 2003], j'ai fouillé les librairies locales pour voir ce qui s'y vendait et ce qui intéressait la communauté locale. En dépit de ces efforts, je n'ai pas pu recueillir d'informations concrètes sur des éléments du passé tel que le niveau de bien-être au cours du passé soviétique, éléments sensibles dont l'interprétation divisait politiquement la communauté. En effet, depuis 2001, avec la victoire aux élections du parti des communistes de Moldavie, pro-russe, la République de Moldavie semblait signer un retour vers le passé communiste.

En même temps, des jeunes de 15 ans croisés quelques jours après mon arrivée m'assuraient qu'ils ne fallait pas «mal juger » leurs parents qui ne savaient pas quel était le sens de l'histoire, qui « étaient encore en URSS » : il suffisait d'attendre qu'eux, la nouvelle génération, aient le droit de vote ${ }^{1}$. Je fus frappée en 2003 par la maturité du discours politique de ces jeunes qui contrastait singulièrement avec l'indifférence politique de leurs parents et les convictions communistes de leurs grands-parents. Mais force est de constater que leurs parents n'avaient alors qu'une seule préoccupation en tête : la subsistance de leurs enfants. C'est pourquoi la plupart avaient fait le choix d'un travail saisonnier à l'étranger et d'un mutisme politique (on note un absentéisme important aux élections durant toute la décennie 2000). Quant aux grands-parents, leur convictions politiques traduisaient un désir de retour à l'ordre et à la force de l'URSs, plus qu'une quelconque conviction idéologique. Qu'est-ce qui explique alors ces choix politiques si différents sous un même toit, le facteur âge avec les responsabilités qu'il entraîne ou l'appartenance à une génération historique différente ? Les choix politiques sont déterminés par les perceptions du passé et du futur qui sont liées à la mémoire sociale et aux idées d'appartenance collective; ils finissent par générer des conflits générationnels. "Ne volez pas notre futur" avait pour titre une série d'articles publiés dans Jurnal de Chisinau en janvierfévrier 2004, fondés sur des interviews relatifs aux choix politiques, et qui polarisait le conflit entre la plus jeune et la plus vieille génération.

La perception du passé est généralement liée à l'âge auquel l'individu fait l'expérience personnelle des événements que traverse son pays. La République de Moldavie a connu des évènements majeurs au tournant du XXI siècle, sur un tempo accéléré : révolution nationale de 1989 (installation de la langue roumaine comme langue nationale, de l'hymne roumain comme hymne national, etc.) ; en 1991 scission de la Moldavie de l'Urss' ${ }^{2}$; mouvements de rue de 1995 et 2002 pour 
le maintien de l'histoire nationale roumaine dans les salles de classe. De plus, la mémoire et le type d'expérience que les citoyens moldaves ont fait de la Roumanie d'entre deux guerres (1918-1940) et de leur appartenance à l'urss (1944-1991) sont également cruciaux et différemment partagés par les individus d'âge différent. C'est pourquoi, en fonction de l'âge, les mémoires "sociales» qui sont aussi générationnelles s'opposent parfois, mènent à des interprétations diverses du contexte social et dictent des lignes de conduites opposées.

J'ai tout vu dans ma vie. J'ai vécu sous les Roumains et sous les soviets, et, regarde, Dieu m'a donné encore des jours à vivre. Et voilà ce que je sais : le mieux était pendant l'urss. Nous avions tout ce dont nous avions besoin et j'étais si jeune! Oui, je sais que les communistes ne nous ramèneront pas l'Urss mais je me sens plus en sécurité avec eux (Jurnal de Chisinau, interview avec un homme de 75 ans).

Son choix politique est donc dicté par une comparaison entre les différentes périodes du passé. Bien qu'il dissocie passé et futur ("les communistes ne nous ramèneront pas l'URss »), il choisit de voter pour le parti politique qui soutient, tout comme lui, une vue positive du passé soviétique. Cela le rassure. La jeune génération qui n'a connu le régime soviétique qu'à travers des ouvrages, lutte uniquement avec les arguments de principe de l'anti-totalitarisme et moins avec leur vécu : "Ne transformez pas notre futur dans le passé horrible que vous avez connu! » ou "J'essaie d'expliquer à mon grand-père que le régime communiste est par définition criminel et antihumain " (Jurnal de Chisinau, 2004). Le vécu du grand-père pèse ici plus lourd dans l'argumentation que la référence livresque du petit-fils, car sa description du passé est plus dense.

Si l'expérience personnelle compte, l'éducation formelle joue aussi un rôle important dans la perception du passé. À la suite de la renaissance identitaire en Moldavie en 1989, les manuels d'histoire et de littérature ont été réécrits. Malgré la perte sur l'échiquier politique des partis pro-roumains quelques années après, depuis le début des années 1990, les enfants ont continué à apprendre à l'école avec des manuels de "Langue et littérature roumaine " et d' "Histoire des Roumains ". Une nouvelle identité est née, différente de celle de leurs parents. Si leurs parents ont vécu la lutte pour l'identité roumaine comme une conquête symbolique qui a eu pour effet de perdre leurs avantages économiques (" on a voulu la langue, l'histoire, et, regarde, maintenant nous mourons de faim » avait coutume de répéter mon hôte communiste de 50 ans), les enfants perçoivent l'identité roumaine et les choix politiques pro-européens comme une évidence. Pour certains, la confrontation entre savoir livresque et discours familiaux est déstabilisante. Dans un film documentaire que j'ai réalisé à l'époque [Heintz et Rus, 2005], la première scène montre une petite fille de dix ans qui fut pendant six mois ma voisine dans un village du nord du pays expliquant qu'elle "parle roumain et cause en moldave ». Devant ce qui semblait une incohérence politique, nous avons demandé des précisions. La réponse de la petite fille tenait encore du domaine de l'évidence : "Parce que c'est comme ça, sur mon manuel c'est écrit : "roumain" ".

Les conflits générationnels en Moldavie portent sur l'interprétation du passé. Le clivage politique dans les années 2000 se situait entre ceux qui considéraient que le régime communiste était une expérience positive, votaient communiste et pro-russe, et ceux qui considéraient que l'appartenance à la Roumanie pendant la période d'entre-deux-guerres était une expérience positive (position conforme au nouveau contenu des manuels d'histoire) et votaient pro- démocratique et pro-roumain/européen.

S'il y a ici une polarisation générationnelle historique, elle est due à l'appui que les partis politiques prennent sur le passé pour justifier leur programme. Il m'a été impossible d'avoir accès pendant cette période aux mémoires individuelles de la période soviétique et roumaine, à travers des récits autres que ceux empruntés aux médias et discours politiques. Tout récit mémoriel était idéologisé : je le reconnaissais aux clichés véhiculés, aux généralités qui ne s'appuyaient jamais sur des faits concrets, et surtout au manque de cohérence entre les épisodes de l'histoire du passé villageois qui m'étaient parfois contés et l'idéologie ouvertement soutenue par le conteur. La mémoire du passé était-elle liée aux intentions de vote ou l'intention de vote était-elle le reflet de la mémoire du passé ? Et pourquoi voter communiste, si ce n'était parce que l'intention de ce vote faisait voir le passé soviétique dans une lumière positive ?

Peut-être qu'ici l'idéologie du changement est finalement secondaire et que ce qui compte est le désir de statu quo, nettement plus important parmi les anciens. En effet, ayant appris à lire en cyrillique, les adultes d'un certain âge ne se sont pas familiarisés avec l'écriture latine nouvellement introduite en 1989, alors que 
leurs enfants l'apprenaient à l'école. Ils n'ont donc pas eu accès aux journaux libres qui paraissent à Chisinau, débattaient du pluripartisme et révélaient les nouveaux choix politiques. En revanche, ils ont continué à regarder la télévision russe comme au temps de l'urss ou à écouter les médias gouvernementaux communistes. Ils n'ont reçu du "changement ", et de plein fouet, que la déchéance économique consécutive à l'accession à l'indépendance de la Moldavie en 1991, qui leur a fait regretter "l'ordre " régnant du temps de l'urss et la libre circulation entre les États de l'Union (dont ils n'avaient pas profité, mais dont les contraintes actuelles empêchent la migration temporaire de leurs enfants).

Il est des cas où les conflits entre générations revêtent des formes plus classiques, la jeune génération défendant le futur sur lequel elle pense avoir le droit de peser, alors que la vieille génération défend son autorité assise sur ses réalisations passées et la preuve qu'elle a donné de ses capacités. Mais les changements sociaux et politiques rapides ont entraîné un déséquilibre entre les générations, déclenchant des conflits politiques au sein des groupes de parenté et brisant les traditions politiques familiales. Le conflit générationnel s'est cristallisé entre les plus jeunes et les plus âgés, mais pas entre les générations successives. La génération du milieu était soit physiquement absente pour cause de migration, soit trop concentrée sur les problèmes de survie économique, ce qui la conduit à se désintéresser de la politique. Il m'a semblé aussi que en l'absence d'un espace public de débat en Moldavie dans les années 2000 [Dungaciu, 2005] qui réduisait ce "débat " à des déclarations contradictoires parallèles, de préférence hors de la présence de son opposant afin d'éviter le conflit ouvert, la jeune génération ne cherchait pas nécessairement à provoquer la génération de ses pères, dont elle dépendait économiquement. Il ne lui restait donc qu'à affronter ses grands-parents. Une autre hypothèse est, qu'en tant que construction historique, les jeunes et la génération médiane appartiennent tous à la même génération historique, - celle des événements de 1989-1991 -, et que, plutôt que la divergence de leurs convictions politiques, l'âge actuel et les contraintes sociales qui lui sont associés induisent des comportements politiques différents.

Que peut faire l'ethnographe dans ce contexte miné par des conflits enlevant toute objectivité aux récits du passé ? Il m'a été difficile de comprendre les particularités des différentes générations parce que je n'avais pas partagé l'intensité de leur vécu historique ${ }^{3}$, ces événements du tournant du siècle qui avaient fini par générer une lassitude au moment où se déroulait mon travail de terrain. Avec les livres d'histoire, j'avais appris que les roumanophones de Moldavie s'étaient battus pour leur identité nationale en 1989, en 1995 et en 2002, mais il m'a fallu du temps pour comprendre que les épisodes successifs au sein de la communauté villageoise, qui divisaient souvent les familles, avaient fini par générer un évitement de toute question historique (car aussi politique). Les récits livresques se concentrent avant tout sur les événements et ne donnent pas la mesure de la densité du changement induite par un trop plein événementiel. J'aurais pu aussi bien passer à côté de cette quasi-évidence, pourtant jamais formulée explicitement par mes interlocuteurs, si je n'avais pas eu comme référence ma précédente expérience de terrain en Roumanie.

\section{"Générations perdues" et "générations attendues" dans le monde du travail en Roumanie}

En 1999-2000, période de crise économique brutale, j'avais effectué à Bucarest quinze mois d'études de terrain relatives aux valeurs du travail dans des organisations relevant du secteur des services. Dix années après la chute de Ceausescu et le rejet inconditionnel du régime communiste (aucune alternative idéologique communiste n'a pu réunir des suffrages depuis 1989 en Roumanie), il n'y avait aucune croissance économique et les conditions de vie s'étaient donc considérablement détériorées depuis les années déjà noires de la fin du régime Ceausescu. Pourtant Bucarest ne connaissait pas le chômage. À 100 dollars par mois, les emplois pullulaient mais ne suffisaient pas pour assurer la survie des familles. Aussi l'emploi principal était-il complété par des emplois privés, souvent non déclarés, rémunérés en fonction du succès de l'opération commerciale, par du travail domestique (notamment en agriculture) ou par le cumul de plusieurs emplois dans le domaine public.

Lors de mon enquête, j'ai travaillé majoritairement aux côtes de jeunes ayant entre 20 et 30 ans (mon âge à l'époque) au sein de deux organisations : une petite entreprise privée et une ONG, les deux employant autour de 40 jeunes dont le turnover était important puisque les conditions de travail et les perspectives de carrière ne paraissaient pas satisfaisantes. Dans les deux organisations, le taux de rémunération horaire 
était supérieur à la moyenne nationale et largement au-dessus de ce que percevaient les employés du secteur public, parfois beaucoup plus qualifiés. Mais les recrutements dans le secteur privé, alors en plein essor, avaient imposé officiellement une limite d'âge stricte à 30 ou 35 ans, afin d'éliminer de ces nouveaux emplois la génération ayant eu une expérience dans le secteur public socialiste. La discrimination en termes d'âge correspondait au désir explicite des employeurs de ne pas embaucher des personnes avec une "mentalité socialiste " - une mesure propre aux années 2000 qui a pris fin officiellement avec l'obligation de ne pas introduire de discrimination envers les plus âgés dans les annonces d'emploi ${ }^{4}$. Si en 1999, il était surtout question d'employer les jeunes dans un secteur privé naissant, l'industrie publique héritée de l'époque socialiste fut démantelée au fur et à mesure et les plus âgés (qui avaient pourtant seulement 40-50 ans au début des années 2000) perdirent définitivement leurs emplois, même mal rémunérés. Aujourd'hui cette tranche d'âge, à l'heure des obligations familiales, est victime de ce handicap pour retrouver un autre emploi dans un secteur privé déjà dirigé par des plus jeunes.

Ainsi, ces pratiques discriminatoires, fondées sur le jugement stéréotypé relatif à l'expérience de travail en contexte socialiste (donc du passé), font naître deux catégories d'employés qui se projettent différemment dans le futur : ceux qui peuvent en envisager un, et ceux qui ne peuvent pas. Leur seul espoir est d'arriver à l'âge de la retraite avant de devenir chômeurs. Sans être même chômeurs, les personnes de 40-50 ans que j'ai pu interviewer en 1999-2000 n'attendaient plus rien, "ne voyaient plus de lumière au bout du tunnel ", n'avaient plus de projet de vie. La résignation et le scepticisme envers les promesses politiques les poussaient à l'absentéisme politique : le taux aux élections locales de juin 2000 fut de $66 \%$ et de $49 \%$ aux élections présidentielles de novembre 2000, suggérant au candidat bucarestois pour l'élection locale au second tour un nouveau slogan: "Qu'est-ce que tu gagnes si tu ne votes pas?". La résignation face au destin a conduit au fatalisme et au catastrophisme dans les années 1999-2000 ; tout événement était le signe d'une fin potentielle: peur de l'éclipse solaire de 1999, peur d'un éventuel tremblement de terre qui aurait pu être déclenché par l'éclipse, peur de la fin du millénaire, peur d'une nouvelle interprétation des prédictions de Nostradamus qui se " confirmera " avec la guerre de Kosovo et le bombardement de Belgrade, capitale "voisine ». Et alors que cette génération de parents obsédés par la survie au quotidien et menacés par la restructuration imminente de leur entreprise broient du noir, leurs enfants vivant sous le même toit réclament un confort occidental, se plaignent de ce que leurs parents n'ont pas choisi des métiers pouvant leur assurer des revenus confortables et abandonnent l'école pour se lancer dans l'entreprenariat. Car les enfants ignorent (tout comme aurait pu le faire l'ethnographe) le fait que leurs parents ont déjà, eux aussi, eut recours à diverses tentatives: des initiatives entrepreneuriales du début des années 1990 avec la Turquie, aux essais de réforme de leurs industries publiques, jusqu'à tenter de se faire embaucher dans des banques et finir par vendre, en faisant du porte-à-porte, les nouveaux produits occidentaux vers le milieu des années 1990.

À la fin de la première décennie, les médias en Roumanie faisaient le constat qu'il existe une " génération perdue » ou " génération sacrifiée » ; ce dernier terme est employé sans état d'âme pour désigner ce qui resulte d'un changement historique considéré comme inéluctable. Depuis 1990 en effet la Roumanie s'est engagée à rejeter les structures économiques du socialisme étatique et à passer à l'économie de marché : dé-collectivisation, privatisation, introduction d'un système régulé par l'offre et la demande et d'un système d'investissements. La transition post-socialiste avec son caractère unidirectionnel est désignée comme un "léninisme inversé " [Gowan, 1995], puisque le sens que devait prendre les transformations n'a jamais été remis en question; en effet, le but du changement - accès à l'économie de marché, démocratie etc. -, à la fois précède et dirige le mouvement. D'une certaine façon, comme dans le cas du léninisme, la nouvelle société fut créée par décret. En 1996, les organismes financiers internationaux craignaient que la dette accumulée depuis 1989 ne soit pas remboursée (l'État n'avait pas de dettes à la fin du régime Ceausescu) et ils obligèrent la Roumanie à accélérer les réformes par l'application d'une "thérapie de choc» - privatisation de ses entreprises inefficaces et restructurations massives - la transition s'accélérant et entraînant appauvrissement, chômage, et instabilité pour les employeurs, considérés comme des "reliques " de l'ancien système. À partir de 2001, la Roumanie connaît un début de croissance économique. Comme pour contrebalancer le cynisme du terme "génération sacrifiée ", utilisé sans discernement, pendant les années de la thérapie de choc, les médias introduisirent au milieu des années 2000 (années de forte croissance économique) son pendant optimiste : "génération attendue ». Naturellement 
cette "génération attendue " existait aussi avant son invention par Cotidianul, un journal influent, mais elle s'est récemment affirmée avec des succès notables jusqu'à obtenir une certaine reconnaissance publique (les ministres de 35-40 ans ne sont plus l'exception en Roumanie). Ironiquement, la génération attendue et la génération sacrifiée partagent un point commun : elles ont ensemble contribué à la révolution de 1989 ou ont mis leurs espoirs dans ce mouvement qui a changé la vie.

Car ce qui frappe dans ce partage générationnel est son aspect arbitraire : à quelques années de distance, les possibilités de vie active changent radicalement et, avec elles, les différences entre style de vie, espoirs et sens du travail. La révolution de 1989 a déclenché un renversement de valeurs. Ceux qui avaient cru aux valeurs communistes se sont sentis perdus, ceux qui étaient en âge de les contester se sont sentis plus forts et ceux qui n'avaient pas encore acquis les nouvelles valeurs citoyennes (parce que trop jeunes) n'ont pas compris l'amplitude du changement qui affectait leurs aînés. Pour déterminer l'âge biologique qui permet de se situer dans une catégorie plutôt que dans une autre, il faudrait faire appel à des considérations psychologiques qui m'échappent, d'autant plus que l'éducation, la position sociale et l'expérience familiale jouent un rôle aussi important que l'âge dans cette catégorisation. Mais empiriquement on constate par exemple que ceux qui sont nés après 1975 (qui n'étaient pas encore au lycée lors de la révolution) n'ont pas compris la transformation historique ("ne savent plus d'où on vient " disent amèrement leurs parents) et sont les produits du capitalisme installé depuis 1989. De la même manière, Yurchak remarque que seulement ceux qui sont nés avant 1970 en URss comprennent le changement qu'a apporté la Perestroïka de Gorbatchev [Yurchak, 1997]. Ainsi, si tous les membres de la nouvelle "génération attendue " partagent le même futur, on remarque à 4-5 années de distance un rapport différent au passé - alors que 4-5 années ne séparent pas une génération au sens classique du terme. En revanche, certains de ses membres ont un rapport au passé similaire à celui de la "génération sacrifiée " avec laquelle ils ne partagent pourtant pas le futur. Cela se traduit en pratique par l'utilisation quasi abusive de l'information donnée par l'âge dans une situation de recrutement, qui est censé en dire long sur les ambitions, espoirs et possibilités du futur employé. Parmi les personnes plus âgées, les différences générationnelles sont moins serrées - les 50-70 ans font ensemble partie des "générations perdues " qui subissent une pression pour quitter l'arène sociale, parce qu'ils ne sont plus employables ou parce que leurs retraites coûtent trop cher à la société. Dans ce cas, les générations historiques prennent le pas sur les générations sociobiologiques et leur analyse fine décèle des différences à peu d'années de distance. Si l'élaboration de "lieux de mémoire " générationnels, dans le sens que leur donne Pierre Nora [1997] est encore à faire, ce n'est qu'une question de temps et les premières tentatives sont déjà apparues [Yurchak, 2005 ; Ernu, 2006].

Plutôt que d'apporter des réponses, mes expériences sur ces deux terrains si proches et pourtant si différents m'amènent à poser des questions. Comment l'anthropologue peut-il ethnographier la diversité sociale rencontrée au présent quand le vécu du passé, auquel il n'a que partiellement accès, l'a façonné ? Si la communauté fondée sur le partage d'un passé et d'un futur commun est si importante, comment une connivence peut-elle s'établir avec celui qui n'a pas vécu nécessairement les événements historiques qui marquent la société ? Suffit-il d' être alerte et critique dans la collecte de récits du passé et du présent, conscient du fait que l'expérience de vie a inégalement touché nos interlocuteurs?

Si la réponse à ces questions reste à venir, l'expérience de ces terrains en transformation rapide m'a appris qu'être sensibilisé au moment historique de collecte de données est indispensable et m'a dicté quelques réflexes indispensables : préciser soigneusement l'année des études de terrain menées, car généraliser historiquement à propos d'une société connaissant un changement rapide est toujours risqué ; ne pas extrapoler historiquement à partir des récits des acteurs, sans s'interroger préalablement sur leur ancrage dans telle ou telle génération historique ; éviter les généralisations qui cachent les conflits internes qui seront le noyau des changements futurs. Ce sont là de simples garde-fous qu'un anthropologue peut dresser une fois que l'enjeu de la temporalité est rendu manifeste. 


\section{NOTES}

1. En avril 2009, ils mèneront effectivement «la Révolte Twitter». Leur rassemblement spontané à caractère révolutionnaire est permis par l'avancement technologique et la popularité de la communication sur réseaux sociaux parmi les jeunes. Suite à cette révolte, de nouvelles élections ont amené des partis démocratiques pro-européens au pouvoir en Moldavie. La balance penche alors du côté démocratique après vingt années d'hésitation politique entre l'Europe et la Russie.

2. L'indépendance de la République de Moladvie proclamée le 27 août 1991 sera par défaut le début de la négation des événements de 1989, car elle annulera l'espoir de réunification avec la Roumanie.

3. Une remarque cependant: il est certain que l'autre raison pour laquelle je n'ai pu mener des interviews ouverts sur les questions de choix politique était le conflit politique roumano-moldave de l'époque, fabriqué en appui de la doctrine moldoveniste promue par le parti communiste au pouvoir. Malgré mon affiliation académique en Allemagne en 2003-2004 lors de mon travail de terrain, j'étais citoyenne roumaine, donc aux yeux de mes interlocuteurs auxquels l'expérience soviétique avait appris la méfiance, j'étais possiblement partie prenante dans ce conflit identitaire. Et si le conflit générationnel était ouvert dans la presse locale sur des questions identitaires, c'est la question identitaire qui primait aux yeux de mes interlocuteurs sur mon appartenance générationnelle. L'explication de certains de mes "échecs " en tant qu'ethnographe exclusivement par l'absence d'un passé commun rencontre ici de possibles limites.

4. Aujourd'hui l'expérience de travail pendant le socialisme s'est réduite grâce à la mise à la retraite anticipée des anciens cadres de l'industrie restructurée, mais il subsiste des traces d'une discrimination envers les plus âgés dans le monde du travail ou du moins d'une promotion explicite des jeunes exclusivement du fait de leur âge.

\section{Références bibliographiques}

Albera Dionigi, 2001, "Terrains minés ", Ethnologie française, XXXI, $1: 5-13$.

Attias-Donfut Claudine et Martine Segalen, 2007 [1998], Grands-parents. La famille à travers les générations, Paris, Odile Jacob.

Burawoy Michael and Katherine Verdery (eds.), 1999, Uncertain Transitions. Ethnographies of Change in the Post Socialist World, Lanham, Rowman \& Littlefield.

Dungaciu Dan, 2005, Moldova Ante Portas [La Moldavie devant les Portes], Bucuresti, Tritonic.

ERnu Vasile, 2006. Nascut in URSS, [Né en URSS], Bucuresti, Polirom.

Fruntasu Iulian, 2003, O istorie etnopolitica a Basarabiei, [Histoire ethnopolitique en Bessarabie], Chisinau, Cartier.

Gowan Peter, 1995, "Neo-Liberal Theory and Practice for Eastern Europe", New Left Review, 2013: 3-60.

Hann Chris. (ed.), 1994, When history Accelerates: Essays in Social Change, Complexity and Creativity, London, Athlone Press.
HeInTz Monica, 2008. Weak State, Uncertain Citizenship, Moldova, Berlin, Peter Lang.

Nora Pierre, 1997, "La génération », in Pierre Nora (dir.), Les Lieux de mémoire, vol. II, Paris, Gallimard : 2975-3015.

Peatrick Anne-Marie (coord.), 1995, "Âges et générations : ordres et désordres Des sociétés aux rythmes du temps", L'Homme, 35, 134, avril-juin.

Peatrick Anne-Marie, 2000, La vie à pas contés, Nanterre, Société d'ethnologie.

Yurchak Alexei, 1997, "The Cynical Reason of Late Capitalism: Power, Pretense and the Anekdot", Public Culture, 9 : 161-188.

YurchaK Alexei, 2005, Everything Was Forever Until It Was No More, Berkeley, California University Press.

\section{| Film}

Heintz Monica et Alin Rus, 2005. Tara de aici, tara de dincolo, [Pays d'ici, pays de là- bas] Film documentaire Max Planck Institute, 58 .

\section{ABSTRACT}

Accelerated history and generational divides. Case studies from Romania and Moldova (1999-2004)

The rapid changes induced by the 1989 revolutions left durable traces among Eastern European populations. Divided in historical generations that do not share a common view on the past and on the future, from "lost generations" to "expected generations", their perception of social life and their inscription into history is reflected in their political, cultural and economic choices. How could an anthropologist describe this current diversity on which lived historical experiences that he did not witness are determinant? The author tackles this question through two field cases conducted at the beginning of the 2000s in Romania and in the Republic of Moldova. Keywords: Generation. Eastern Europe. Work. Temporalities. Sensitive fieldwork. 